coloproctology $2010 \cdot 32: 78-78$

DOI 10.1007/s00053-010-0062-2

๑) Urban \& Vogel 2010

\title{
Chronik \\ der Deutschen Gesellschaft für Koloproktologie
}

Die Gesellschaft wurde am 21. November 1990 in Düsseldorf als gemeinnütziger Verein (e.V.) gegründet. Ziel der Gesellschaft (DGK) ist die Förderung der wissenschaftlichen Forschung auf dem Gebiet der Koloproktologie und ihrer Grenzgebiete. Insbesondere der interdisziplinäre Charakter der Koloproktologie soll durch die Zusammensetzung der Mitglieder, des Präsidiums sowie der Tagungsprogramme zum Ausdruck kommen.

Gründungsmitglieder waren:

R. Alpers, W. Brühl, P. Forstmann, Th. Hager, C. Junghans, J.J. Kirsch, J. Lill, G. Mahlke, J. Meier zu Eissen, H. Mlitz, F. Raulf, H. Schüler, V. Wienert und O. Zenner.

Zum ersten Präsidenten wurde Th. Hagergewählt. Diese Gründung wurde wesentlich beeinflusst durch fachliche und finanzielle Unterstützung des damaligen Berufsverbandes der Proktologen Deutschlands (BCD) und den persönlichen, großen Einsatz von P. Forstmann und Th. Hager.

Seit 1991 veranstaltet die DGK ihren Jahreskongress - die „Koloproktologie-Tage" - gemeinsam mit dem Berufsverband der Coloproktologen Deutschlands in Bad Homburg. Ab 1995 schloss sich die Arbeitsgemein- schaft für Coloproktologie (CACP) der Deutschen Gesellschaft für Chirurgie als Mitveranstalter an. Wegen zunehmender Resonanz erfolgte 1997 der Umzug nach München. Mittlerweile wurde der Jahreskongress von regelmäßig über 400 Teilnehmern besucht. 2002 hat sich auch die Arbeitsgemeinschaft fürColoproctologie(ACP)Österreichs dem Kongress angeschlossen. 2003 ist die Integration der Schweizerischen Arbeitsgruppe für Koloproktologie, der Sektion für Proktologie der DGVS und AG Proktologie der Deutschen Dermatologischen Gesellschaft gelungen. Im Jahre 2005 hat sich mit der Gesellschaft für Neurogastroenterologie und Motilität eine weitere wissenschaftliche Gesellschaft dem Jahreskongress angeschlossen. Im Jahr 2006 wurde erstmals die „Schallmauer“ von 600 Kongressteilnehmern überschritten.

2001 wurde die DGK in die Arbeitsgemeinschaft wissenschaftlicher medizinischer Fachgesellschaften Deutschlands (AWMF) aufgenommen. Gemeinsam mit der Deutschen Gesellschaft für Viszeralchirurgie - vertreten durch die CACP - vergibt die DGK seit 2007 an qualifizierte Kliniken das Zertifikatfür chirurgische Kompetenz- und ReferenzZentren.

\section{Bisheriges Präsidium:}

\begin{tabular}{|c|c|c|c|c|}
\hline Jahr & Präsident & Vizepräsident & Geschäftsführer & Schatzmeister \\
\hline 1990 & Prof. Th. Hager & Prof. V. Wienert & Dr. P. Forstmann & Dr. Dr. R. Alpers \\
\hline 1991 & Prof. Th. Hager & Prof.V.Wienert & Dr. P. Forstmann & Dr. Dr. R. Alpers \\
\hline 1992 & Prof. G. Lux & Prof. V. Wienert & Prof. Th. Hager & Dr. Dr. R. Alpers \\
\hline 1993 & Prof.G. Lux & Prof. V.Wienert & Prof. Th. Hager & Dr. J. Lill \\
\hline 1994 & Prof. V.Wienert & Prof. R. Winkler & Prof. Th. Hager & Dr. J. Lill \\
\hline 1995 & Prof. V. Wienert & Prof. R. Winkler & Prof. Th. Hager & Dr. J. Lill \\
\hline 1996 & Prof. R. Winkler & Prof. H. Egger & Prof. Th. Hager & Dr. J. Lill \\
\hline 1997 & Prof. R. Winkler & Prof. H. Egger & Prof. Th. Hager & Dr.J. Lill \\
\hline 1998 & Prof. H Egger & Prof. W. Heitland & Prof. Th. Hager & Dr.J. Lill \\
\hline 1999 & Prof. H. Egger & Prof. W. Heitland & Prof. Th. Hager & Dr.J. Lill \\
\hline 2000 & Prof.W. Heitland & Prof. W. Hartschuh & Prof. Th. Hager & Dr.J. Lill \\
\hline 2001 & Prof. W. Heitland & Prof. W. Hartschuh & Prof. Th. Hager & Dr.J. Lill \\
\hline 2002 & Prof. W. Hartschuh & Dr. G. Pommer & Prof. Th. Hager & Dr.J.Lill \\
\hline 2003 & Prof. W. Hartschuh & Dr. G. Pommer & PD Dr. A. Herold & PD Dr. T. Schiedeck \\
\hline 2004 & Dr. G. Pommer & Prof. Dr. K-W. Jauch & PD Dr. A. Herold & PD Dr. T. Schiedeck \\
\hline 2005 & Dr. G. Pommer & Prof. Dr. K-W. Jauch & PD Dr. A. Herold & PD Dr. T. Schiedeck \\
\hline 2006 & Prof. Dr. K.-W. Jauch & Prof. Dr. F. Hartmann & Prof. Dr. A. Herold & PD Dr. T. Schiedeck \\
\hline 2007 & Prof. Dr. K.-W. Jauch & Prof. Dr. F. Hartmann & Prof. Dr. A. Herold & PD Dr. T. Schiedeck \\
\hline 2008 & Prof. Dr. F. Hartmann & Prof. Dr. R. Arbogast & Prof. Dr. A. Herold & Prof. Dr. T. Schiedeck \\
\hline 2009 & Prof. Dr. F. Hartmann & Prof. Dr. R. Arbogast & Prof. Dr. A. Herold & Prof. Dr. T. Schiedeck \\
\hline 2010 & Prof. Dr. R. Arbogast & Prof. Dr. E. Stange & Prof. Dr. A. Herold & Prof. Dr. T. Schiedeck \\
\hline
\end{tabular}

